\title{
Membrane Engineering via Trans Unsaturated Fatty Acids Production Improves Escherichia coli Robustness and Production of Biorenewables
}

\author{
Zaigao $\operatorname{Tan}^{1}$ \\ Jong Moon Yoon ${ }^{1}$ \\ David R. Nielsen ${ }^{2}$ \\ Jacqueline V. Shanks ${ }^{1}$ \\ Laura R. Jarboe ${ }^{1 *}$
}

${ }^{1}$ Department of Chemical and Biological Engineering, Iowa State University, Ames, Iowa, 50011, USA

${ }^{2}$ Chemical Engineering, School for Engineering of Matter, Transport, and Energy, Arizona State University, Tempe, Arizona, 85287-6106, USA

*Corresponding author: Laura R. Jarboe, Department of Chemical and Biological Engineering, 4134 Biorenewables Research Laboratory, Iowa State University, Ames, IA, USA, 50011, +1-515-294-2319, ljarboe@iastate.edu 


\begin{abstract}
Constructing microbial biocatalysts that produce biorenewables at economically viable yields and titers is often hampered by product toxicity. For production of short chain fatty acids, membrane damage is considered the primary mechanism of toxicity, particularly in regards to membrane integrity. Previous engineering efforts in Escherichia coli have attempted to increase membrane integrity, with the goal of increasing fatty acid tolerance and production, with mixed results. Herein, a novel approach was used to reconstruct the E. coli membrane by enabling production of a novel membrane component. Specifically, trans unsaturated fatty acids (TUFA) were produced and incorporated into the membrane of E. coli MG1655 by expression of cis-trans isomerase (Cti) from Pseudomonas aeruginosa. While the engineered strain was found to have no increase in membrane integrity, a significant decrease in membrane fluidity was observed, meaning that membrane polarization and rigidity were increased by TUFA incorporation. As a result, tolerance to exogenously added octanoic acid and octanoic acid production were both increased relative to the wild-type strain. This membrane engineering strategy to improve octanoic acid tolerance was found to require fine-tuning of TUFA abundance. Besides improving tolerance and production of carboxylic acids, TUFA production also enabled increased tolerance in E. coli to other bio-products, e.g. alcohols, organic acids, aromatic compounds, a variety of adverse industrial conditions, e.g. low $\mathrm{pH}$, high temperature, and also elevated styrene, another versatile bio-chemical product. TUFA permitted enhanced growth due to alleviating bio-product toxicity, demonstrating the general effectiveness of this membrane engineering strategy towards improving strain robustness.
\end{abstract}

Keywords: trans unsaturated fatty acids (TUFA); tolerance; membrane fluidity; membrane integrity; carboxylic acids production 


\section{INTRODUCTION}

Engineering of microbial catalysts for the production of fuels and chemicals is a promising, biorenewable alternative to current petroleum-based methods $(1,2)$. A variety of microorganisms have been engineered for production of biofuels, bulk chemicals, and value added chemicals (3-7). However, toxicity of biorenewable products to the host strain often limits the strain performance $(8,9)$. For instance, ethanol-producing Escherichia coli grows poorly in the presence of $35 \mathrm{~g} \mathrm{~L}^{-1}$ ethanol and less than $10 \%$ of cells survive when exposed to $100 \mathrm{~g} \mathrm{~L}^{-1}$ of ethanol (10). Clostridia produces n-butanol during ABE fermentation, but does not grow when challenged with $2 \%(\mathrm{v} / \mathrm{v}) \mathrm{n}$-butanol (11). The bulk industrial chemical styrene can be produced by engineered microbes, but significantly inhibits the growth of the producer strain at a concentration of only $300 \mathrm{mg} \mathrm{L}^{-1}(5)$.

Inhibitory concentrations of products can cause a variety of detrimental effects to production strains. Arguably, the most frequently described effect is membrane damage, often considered as a general mechanism of toxicity (12-16). As the primary architecture of the cell, the membrane plays important roles in transport, energy exchange and protection from infection (17). Alcohols, such as ethanol, can inhibit cell growth by causing membrane leakage (16) and longer chain alcohols, such butanol, also cause leakage of important metabolites and fluidize the cell membrane (12). Some organic acids, e.g. acetic acid, also induce membrane leakage (18).

Carboxylic acids, which can serve as catalytic precursors for a variety of chemicals (19), are broadly useful as lubricants, preservatives, fuels and other applications $(20,21)$. In recent years, E. coli and Saccharomyces cerevisiae have both been engineered to produce carboxylic acids at high titer, productivity and yield (4, 20, 22-29). However, as with other biorenewable chemicals , high concentrations of carboxylic acids inhibit the growth of the host strain and decrease the performance of engineered strains $(14,30,31)$. Carboxylic acids can cause a variety of detrimental effects, including membrane damage, cellular expansion, intracellular acidification, and disruption of amino acid pools (13-15, 20, 24, 31-33). Among these various effects, membrane damage is typically considered as the primary cause of carboxylic acid toxicity (13, $15,32,34,35)$. Multiple studies have reported membrane leakage of $E$. coli during fatty acid production. Lennen et al. reported that the membrane leakage significantly increased after induction of fatty acid production and cell viability decreased by $85 \%$ relative to the control strain (13). Lennen's transcriptome analysis of strains during fatty acid production showed that 
even early in the production phase when titers were still below $100 \mathrm{mg} / \mathrm{L}$, genes known to be activated in response to membrane damage had increased expression relative to the non-producing control (13). Our own prior results also showed that both exogenously-added octanoic acid (C8) and endogenously-produced long-chain fatty acids (C14+C16) significantly increased membrane leakage (15).

To this end, construction of a "stronger" membrane may increase the tolerance of microbial biocatalysts to carboxylic acids, with the goal of further increasing carboxylic acids production. Lennen et al. showed that expressing a thioesterase, which prevents medium chain unsaturated acyl-ACPs from being incorporated into the membrane, decreased unsaturated fatty acid content in the membrane and thus increased cell integrity during fatty acid production (32). Although this was effective in decreasing membrane leakage, no increase in production was observed (32). Sherkhanov et al. found that deletion of the pathway responsible for incorporating medium chain fatty acids into the membrane increased the average length of membrane lipids, alleviated the toxicity of fatty acids and improved fatty acid production by $20 \%$ (35). In our prior study, E. coli was evolved for tolerance of exogenous octanoic acid. The evolved strain not only showed increased tolerance of fatty acids, but also improved fatty acid production and even increased butanol tolerance. These changes in tolerance and production were accompanied by an increase in the average membrane lipid length and decreased membrane leakage (34).

These results demonstrate the possibility of improving carboxylic acids tolerance and/or production by modification of the microbial cell membrane, specifically by altering the chain length or degree of saturation of the membrane components in order to increase membrane integrity and mitigate leakage $(13,15,32,34,35)$. However, some intriguing possibilities remain. First, are there other routes for changing membrane lipid composition? Second, can other membrane characteristics be addressed to improve tolerance? Finally, besides carboxylic acids, could membrane engineering exhibit general effects in increasing microbial robustness in the context of other bio-products and/or severe growth conditions?

In order to answer these questions, we introduced a non-native component into the $E$. coli membrane. Specifically, trans unsaturated fatty acids (TUFA) were synthesized by and incorporated into the membrane of E. coli via expression of the Cti enzyme from Pseudomonas aeruginosa. In contrast to other engineering strategies $(32,35)$, our engineered strain showed no change in the membrane phospholipid length or integrity. Instead, there was a decrease in 
membrane fluidity. This engineered strain displayed increased tolerance to octanoic acid and increased octanoic acid titer. In addition, we found that TUFA production also enabled increased tolerance to other biorenewables as well as a variety of adverse environmental conditions of industrial relevance.

\section{MATERIALS AND METHODS}

2.1 Strains and Plasmids: All plasmids and strains constructed in this study are listed in Table 1. Two forms of $c t i$, intact $c t i$ with the native signal peptide sequence and a truncated $c t i$ with the PelB signal peptide sequence, were regulated by three different promoters with varying strengths (M1-12, M1-37, M1-93) $(36,37)$ and then inserted individually into the E. coli MG1655 genome. For octanoic acid production the $f a d D$ gene was deleted to prevent fatty acid degradation and strains were transformed with the pJMYEEI82564 plasmid (34) harboring thioesterase (TE10) from Anaerococcus tetradius (38). One-step recombination method (FLP-FRT) was employed in this study (39).

Table 1. Plasmids and strains used in this study.

Plasmids/Strains

Genetic characteristics

Resource

Plasmids
pJMYEEI82564
pKD3
pET22b
pET22b-cti
pTpal-fdc
Strains
Pseudomonas
aeruginosa
MG1655
Control
M1-12-Pacti
M1-37-Pacti
M1-93-Pacti
M1-12-PeB-Pacti
M1-37-PeB-Pacti
M1-93-PeB-Pacti
ldhAD+TE10

pTrc-EEI82564 thioesterase (TE10) from Anaerococcus tetradius, Amp ${ }^{\mathrm{r}}$ (34)

Containing FRT-Cat-FRT fragment, $\mathrm{Cm}^{\mathrm{r}}$

Expression vector with $p e l B$ coding sequence, $A m p^{r}$

Novagen

pET22b, FRT-Cat-FRT DNA fragment from pKD3 and $c t i$ gene from $P$. This study aeruginosa cloned into pET22b, $\mathrm{Amp}^{\mathrm{r}} \mathrm{Cm}^{\mathrm{r}}$

$P A L$ of $A$. thaliana and $F D C$ of $S$. cerevisiae inserted into pTrc99a (40) plasmid, Amp ${ }^{\mathrm{r}}$

Pseudomonas aeruginosa wild type

Shao lab, ISU

E. coli $\mathrm{K}-12$ wild type

This lab

MG1655, ldhA:: FRT-Cat-FRT

This study

MG1655, ldhA:: FRT-Cat-FRT, M1-12-Pacti

This study

MG1655, ldhA:: FRT-Cat-FRT, M1-37-Pacti

This study

MG1655, ldhA:: FRT-Cat-FRT, M1-93-Pacti

This study

MG1655, ldhA:: FRT-Cat-FRT, M1-12-PelB-Pacti

This study

MG1655, ldhA:: FRT-Cat-FRT, M1-37-PelB-Pacti

This study

MG1655, ldhA:: FRT-Cat-FRT, M1-93-PelB-Pacti

This study

Control, $\Delta f a d D$, pJMYEEI82564

This study 
2.2 Growth Rate Characterization: Specific growth rate (/hr) was calculated by fitting the equation $\mathrm{OD}=\mathrm{OD}_{0} \mathrm{e}^{\mathrm{t}}$ over the duration of the exponential growth phase. OD was measured at $550 \mathrm{~nm}$ and all estimated values had an $\mathrm{R}^{2}$ of at least 0.95 .

2.3 Strain Tolerance Characterization: All tolerance experiments were implemented in $50 \mathrm{~mL}$ MOPS 2.0\% (w/v) dextrose medium (41) in $250 \mathrm{~mL}$ baffled flasks at $200 \mathrm{rpm}$ and initial $\mathrm{pH}$ of 7.0. Tolerance to octanoic acid was assessed at $30^{\circ} \mathrm{C}$, high temperature tolerance was assessed at $42{ }^{\circ} \mathrm{C}$, and all other tolerance experiments were performed at $37^{\circ} \mathrm{C}$.

2.4 Membrane Characterization: Membrane integrity was analyzed by SYTOX green staining and membrane fluidity was measured by using 1,6-diphenyl-1,3,5-hexatriene (DPH). Membrane composition was measured by a modified Bligh and Dyer method. All of these analyses were performed as previously described (15). The two-tailed t-test method was employed to analyze the statistical significance of all data in this study.

2.5 Batch Fermentation for Carboxylic Acid Production: Individual colonies were selected from Luria Broth (LB) plates with $100 \mathrm{mg} / \mathrm{L}$ ampicillin, inoculated into $3 \mathrm{ml}$ of LB liquid medium with $100 \mathrm{mg} / \mathrm{L}$ of ampicillin for $4 \mathrm{hrs}$. Then, $1 \mathrm{ml}$ of culture was added to $50 \mathrm{ml}$ MOPS $2.0 \%$ dextrose medium with $100 \mathrm{mg} / \mathrm{L}$ of ampicillin at $30^{\circ} \mathrm{C}, 200 \mathrm{rpm}$ overnight for seed culture preparation. Mid-log phase seed culture was collected, resuspended with fresh MOPS 2.0\% dextrose medium and transferred into $300 \mathrm{ml}$ MOPS $2.0 \%$ dextrose medium with $100 \mathrm{mg} / \mathrm{L}$ of ampicillin and $1 \mathrm{mM}$ of isopropyl- $\beta$-D-thiogalactopyranoside (IPTG) in $500 \mathrm{ml}$ fermentor (INFORS HT) at the final concentration of $\mathrm{OD}_{550} \sim 0.1$. Cultures were grown at $30^{\circ} \mathrm{C}$, and the $\mathrm{pH}$ was maintained at 7.0 by addition of $2 \mathrm{M} \mathrm{KOH}$. Air flow rate was maintained at $0.3 \mathrm{~L} / \mathrm{min}$ initially. The dissolved oxygen (DO) level was set over $40 \%$ and controlled by changing the stirring speed to a maximum of $600 \mathrm{rpm}$.

2.6 Determination of Carboxylic Acid Titers: For total fatty acids titer measurement, $400 \mu \mathrm{L}$ of culture was extracted and quantified as previously described (42). For free fatty acids analysis, the fermentation broth was centrifuged and same volume of supernatant was quantified (42). 2.7 Styrene Production: The pTpal-fdc plasmid (40) harboring PAL2 from Arabidopsis thaliana and FDCl from S. cerevisiae was transformed into E. coli. A single colony was selected and 
inoculated into $5 \mathrm{ml}$ of LB liquid medium with $100 \mathrm{mg} / \mathrm{L}$ of ampicillin. After incubation at $32{ }^{\circ} \mathrm{C}$, $220 \mathrm{rpm}$ overnight, this seed culture was added to $50 \mathrm{ml}$ of MOPS $2.0 \%$ dextrose with $100 \mathrm{mg} / \mathrm{L}$ of ampicillin, $1 \mathrm{~g} / \mathrm{L} \mathrm{L}$-phenylalanine, $0.5 \mathrm{mM}$ of IPTG at the final concentration of $\mathrm{OD}_{550} \sim 0.1$ in $250 \mathrm{ml}$ shake flasks. This culture was incubated at $32{ }^{\circ} \mathrm{C}, 220 \mathrm{rpm}$ for $72 \mathrm{hrs}$. Styrene was quantified as described by McKenna et al. (5)

\section{RESULTS}

\subsection{Enabling TUFA production and incorporation in the $E$. coli membrane}

Phospholipids are the primary structural constituent of the cell membrane (43). A phospholipid molecule generally consists of a hydrophilic phosphate head and two hydrophobic fatty acids tails (44). There are two different forms of fatty acids in phospholipids: saturated fatty acids (SFA), e.g. C16:0, and unsaturated fatty acids (UFA), e.g. C16:1. UFAs can be further divided into cis unsaturated fatty acids (CUFA) and TUFA. Although CUFA and TUFA with same carbon numbers have identical average chain length, their conformations are distinct. CUFAs bend at an angle of $\sim 30^{\circ}$ at the $\Delta 9$ double bond position while TUFAs are straight, resembling the structure of SFA (Fig 1A) (45).

Packing of CUFAs in the membrane will form a disorderly membrane and increase its fluidity (46). CUFAs can, however, be converted to the corresponding TUFAs, as demonstrated by several Pseudomonas and Vibrio strains (47). The conformational change enabled by this process, known as homeoviscous adaptation $(45,47-49)$, increases the membrane phase transition temperature, thereby improving survival in severe environments, including exposure to toxic organic solvents. However, model biorenewables-producing microorganisms, such as $E$. coli and S. cerevisiae, have not been reported to naturally produce TUFAs. The enzyme responsible for catalyzing the conversion of CUFAs to TUFAs is Cis-trans isomerase (Cti). Therefore, we proposed that enabling cis-trans isomerization in model strains via expression of the Cti enzyme might increase robustness in adverse conditions typically faced in a bioproduction environment.

Packing of CUFAs in the membrane will form a disorderly membrane and increase its fluidity (46). CUFAs can, however, be converted to the corresponding TUFAs, as demonstrated by several Pseudomonas and Vibrio strains (47). The conformational change enabled by this process, known as homeoviscous adaptation $(45,47-49)$, increases the membrane phase 
transition temperature, thereby improving survival in severe environments, including exposure to toxic organic solvents. However, model biorenewables-producing microorganisms, such as $E$. coli and $S$. cerevisiae, have not been reported to naturally produce TUFAs. The enzyme responsible for catalyzing the conversion of CUFAs to TUFAs is Cis-trans isomerase (Cti). Therefore, we proposed that enabling cis-trans isomerization in model strains via expression of the Cti enzyme might increase robustness in adverse conditions typically faced in a bioproduction environment.

A

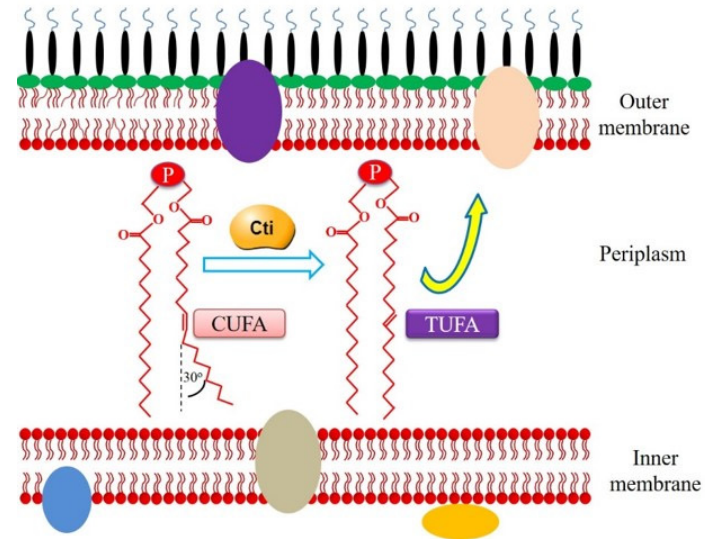

B

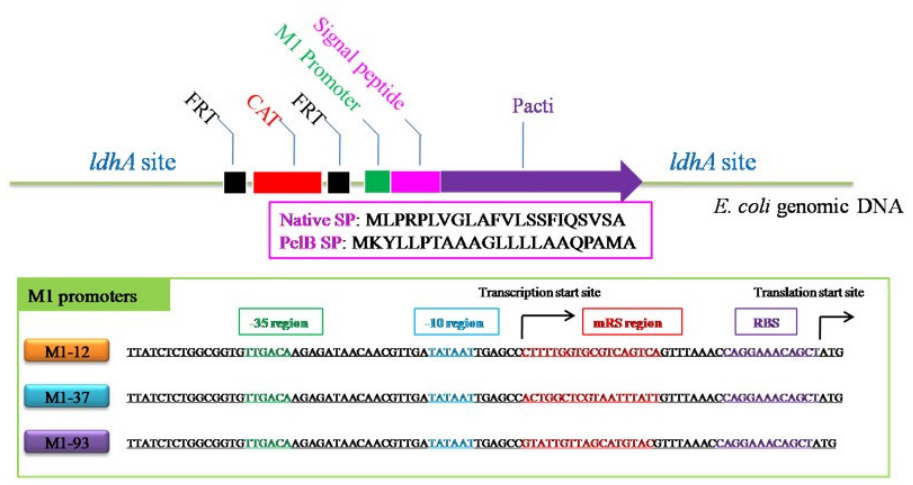

Figure 1: Functional characteristics and expression of cis-trans isomerase (Cti) in E. coli.

(A) Conversion of cis unsaturated phospholipid fatty acids (CUFA) to trans unsaturated phospholipid fatty acids (TUFA) by Cti. Mature Cti is located in the periplasmic space of Pseudomonas and Vibrio strains. CUFA will form a kink with an angle of $30^{\circ}$ at double bond position $(\Delta 9)$. Cti changes the configuration of CUFA at the $\Delta 9$ position to form the corresponding TUFA without a kink.

(B) Integration of cti from Pseudomonas aeruginosa (Pacti) into genomic DNA of E. coli MG1655 at the ldhA (lactate dehydrogenase) site using FRT-CAT-FRT as selection marker. Two kinds of signal peptide (SP), native SP and PelB SP were used to transport Cti across the inner membrane. Three different promoters (M1-12, M1-37, M1-93) were employed to regulate the expression of $c t i$. The three promoters have the same -35 region (green), -10 region (cyan) and RBS region (purple), while the mRS (ㅍNA stabling) regions (red) are distinct.

Mature Cti isomerase is located in the periplasmic space of Pseudomonas and Vibrio sp and a signal peptide is required for its transport across the cytoplasmic membrane (50). Although Cti contains a native signal peptide, we postulated that it might not be correctly transported in $E$. coli. Thus, two different strategies for expression of $\underline{P}$. aeruginosa cis-trans isomerase (Pacti) in E. coli were employed: 1) Expression of intact Pacti with its native signal peptide; 2) Substitution of the native signal peptide with the PelB signal peptide (Fig 1B). In addition, for 
the sake of tunable expression of Pacti, three different constitutive promoters, M1-12, M1-37, M1-93 $(36,37)$, of which the mRNA stabling region (mRS) sequences are different, were employed. Thus, six Pacti engineered E. coli strains with different combinations of promoters and signal peptides were constructed (Table 1).

Strains expressing Pacti with either its native signal peptide or the PelB signal peptide were found to contain TUFA in the membrane (Fig 2A), meaning that in both cases, Cti was able to be transported to the periplasmic space.

\subsection{Production of TUFA increases $E$. coli octanoic acid tolerance}

In order to investigate the effect of TUFA production on E. coli tolerance of octanoic acid, the growth of our six engineered strains was assessed in the presence of three different concentrations of octanoic acid. Note that there was no significant difference $(\mathrm{P}<0.05)$ in the specific growth rate between all Pacti engineered strains and the control strain in the absence of octanoic acid (Fig 2B). Consistent with our hypothesis, most of the Pacti engineered strains were found to have increased tolerance to octanoic acid compared with the control strain (Fig 2B). For instance, in the presence of $10 \mathrm{mM}$ octanoic acid, the specific growth rate of M1-12-Pacti and M1-93-Pacti increased $12 \%(\mathrm{P}=0.007)$ and $10 \%(\mathrm{P}=0.008)$ over the control strain $\left(0.308 \mathrm{~h}^{-1}\right)$, respectively. This trend continues under higher concentrations of octanoic acid. In the presence of $20 \mathrm{mM}$ octanoic acid, the specific growth rate of M1-12-Pacti and M1-93-Pacti strains were $13 \%(\mathrm{P}=0.048)$ and $12 \%(\mathrm{P}=0.078)$ higher than the control strain $\left(0.227 \mathrm{~h}^{-1}\right)$.

However, not all Pacti engineered strains had significantly increased octanoic acid tolerance. Strain M1-37-Pacti had specific growth rates similar to the control strain in the presence of 10 or $20 \mathrm{mM}$ octanoic acid (Fig 2B). Further characterization showed that the molar ratio of TUFA/CUFA in M1-37-Pacti is only $0.055 \pm 0.008$, which although higher than that in the control strain $(\sim 0)$, is lower than that in the other engineered strains (Fig $2 \mathrm{C})$. This suggests that the lack of increased octanoic acid tolerance of M1-37-Pacti results from the lower TUFA/CUFA ratio.

Consistent with these findings, we found that there is a dual relationship between the TUFA/CUFA ratio and the specific growth rate increases in the presence of $10 \mathrm{mM}$ octanoic acid (Fig 2C). For instance, the TUFA/CUFA ratio in M1-12-Pacti is $0.078 \pm 0.01$, and there was a $12 \%$ increase in specific growth rate relative to the control strain. However, ratios larger than 0.09 were associated with decreased specific growth rate. For instance, the TUFA/CUFA ratio in M1-12-PelB-Pacti is $0.16 \pm 0.02$, which is about 2-fold higher than the ratio of M1-12-Pacti, but 
the specific growth rate of M1-12-PelB-Pacti strain only increased by $5 \%$ relative to control strain. These findings suggest that careful tuning of the relative abundance of the TUFA is required in order to positively impact strain robustness.

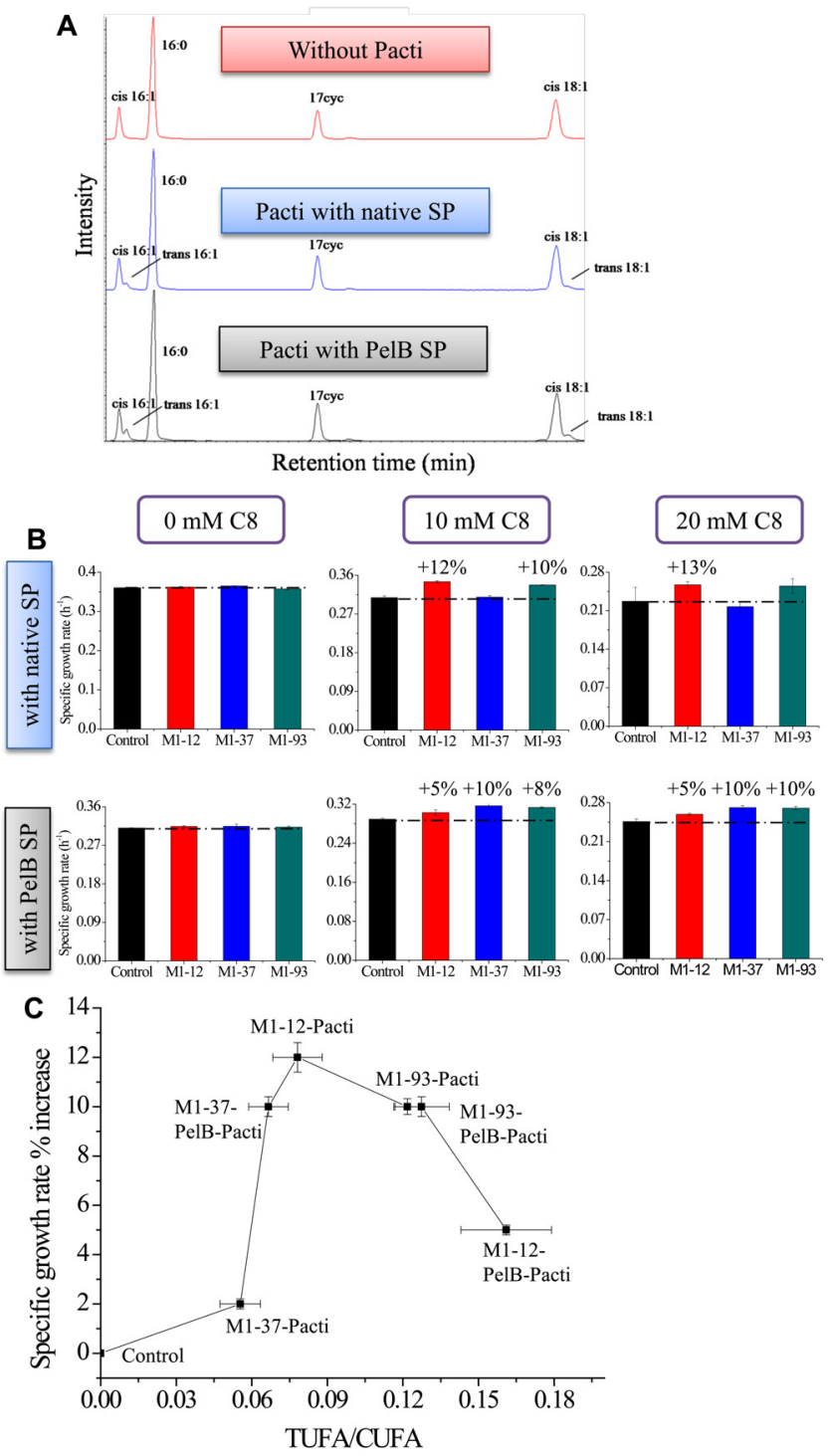

Figure 2: Appropriately tuned production of TUFA enables increased octanoic acid tolerance.

(A) Membrane fatty acids profile of strains without Pacti (upper), Pacti with the native signal peptide (SP, middle), and a truncated Pacti with the PelB SP (lower). Either using native SP or PelB, Pacti could be transported into periplasm of $E$. coli and catalyze TUFA formation (e.g. trans 16:1, trans 18:1). (B) Specific growth rate changes of $c t i$ engineered strains with different promoters (M1-12, M1-37, M1-93) under varying concentrations of octanoic acid (C8) conditions. Strains were grown in MOPS $+2 \%$ dextrose shake flasks at 200 rpm $30{ }^{\circ} \mathrm{C}$ with an initial $\mathrm{pH}$ of 7.0. (C) Relationship between ratio of TUFA/CUFA and specific growth rate \% increase under $10 \mathrm{mM} \mathrm{C} 8$ condition. Values are the average of three biological replicates with error bars indicating one standard deviation. $\%$ increase values are only shown for differences that were deemed statistically significant $(\mathrm{P}<0.05)$ using a two-tailed t-test.

\subsection{TUFA-producing strains have increased membrane rigidity}

The composition of the membrane contributes to its overall physical properties, such as membrane integrity and fluidity. Given that the M1-12-Pacti strain exhibits the most prominent increase in octanoic acid tolerance, we chose this strain for membrane characterization under 10 mM C8 stress.

In previous studies, altering the saturated/unsaturated $(\mathrm{S} / \mathrm{U})$ ratio in the membrane or 
decreasing the incorporation of shorter lipids into the membrane have been the main routes used to address membrane damage $(32,51,52)$. However, here we found that membrane $\mathrm{S} / \mathrm{U}$ ratio was not changed in our TUFA-producing strains. For instance, the S/U ratio in M1-12-Pacti was not significantly different from the non-TUFA producing control strain (Fig $3 \mathrm{~A}$ ). In addition, the average lipid length in M1-12-Pacti was almost identical to that of the control strain (Fig 3A), suggesting that the membrane "thickness" was not affected by TUFA production and incorporation.

Membrane leakage has previously been observed in fatty acid producing strains, and efforts to improve membrane integrity have been effective in increasing viability $(13,32)$. However, membrane integrity of M1-12-Pacti was not enhanced by TUFA production and incorporation (Fig 3B). The fact that carboxylic acid tolerance was increased without increasing membrane integrity is distinct from previously-described strains with increased carboxylic acid tolerance $(32,34)$.

In contrast to the lack of change in membrane integrity, we found the membrane polarization value of M1-12-Pacti $(0.386 \pm 0.003)$ to be increased by $10.3 \%(\mathrm{P}<0.01)$ relative to the control strain $(0.35 \pm 0.01)$ (Fig 3B). This means that the membrane fluidity of the engineered strain was decreased when TUFA production was enabled.

A

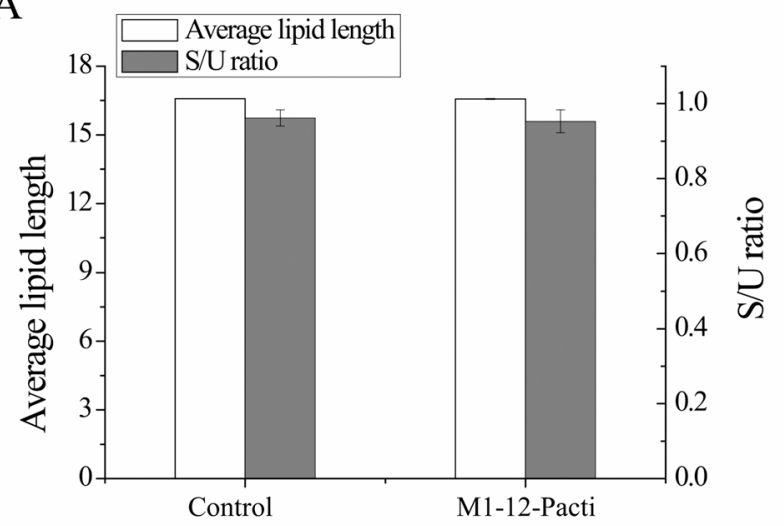

B

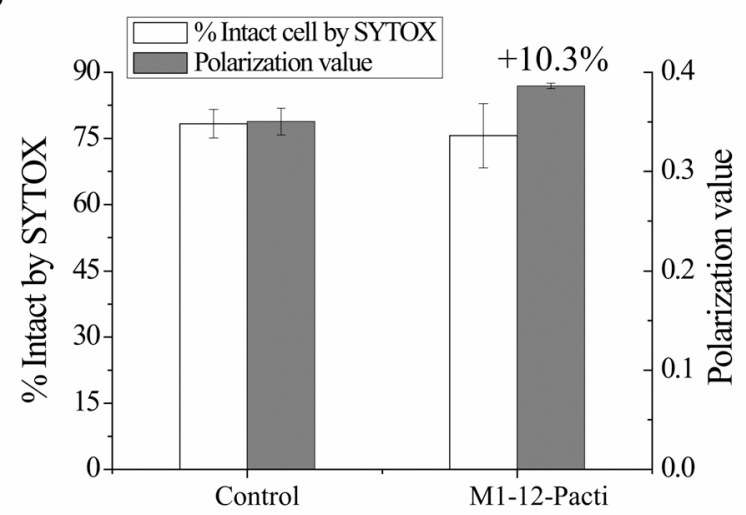

Figure 3: TUFA production primarily alters membrane rigidity, not integrity. All characteristics were assessed after growth in the presence of $10 \mathrm{mM} \mathrm{C} 8$ at $30{ }^{\circ} \mathrm{C}$, initial $\mathrm{pH}$ 7.0. (A) Average lipid length and saturated/unsaturated $(\mathrm{S} / \mathrm{U})$ ratio of $\mathrm{M}-12$-Pacti engineered strain and control strain. No significant changes of either metric was observed. (B) Percentage of intact cells, assessed using SYTOX Green, and membrane polarization, assessed using DPH, of M1-12-Pacti and control strain. \% increase values are only shown for differences that were deemed statistically significant $(\mathrm{P}<0.05)$ using a two-tailed $\mathrm{t}$-test. Values are the average of three biological replicates with error bars indicating one standard deviation. 


\subsection{TUFA production increases octanoic acid production}

Since the engineered TUFA-producing strains demonstrated increased specific growth rate when challenged with exogenously supplied octanoic acid, we tested whether this increased tolerance also enabled improved octanoic acid production. Specifically, the M1-12-Pacti strain was engineered for octanoic acid production by deletion of $f a d D$ and expression of a plasmid-encoded thioesterase, resulting in strain M1-12-PactiD+TE10. Analogous manipulations were also performed on the control strain to obtain the corresponding non TUFA-producing ldhAD+TE10 strain.

When characterized in minimal medium, the engineered strain had a shorter lag than the engineered strain, both in terms of growth and glucose consumption (Fig 4A). By 72 hours, the two strains had reached similar optical density values. Starting at the 48 hour timepoint, the TUFA-producing strain had increased titers of octanoic acid and total fatty acids relative to the control strain (Fig 4B). The final octanoic acid titer of the engineered strain was $41 \%$ higher $(43.7 \mathrm{mg} / \mathrm{L})$ than the control $(31.0 \mathrm{mg} / \mathrm{L})$ and total fatty acids were $35 \%$ higher $(82.1 \mathrm{mg} / \mathrm{L})$ than the control $(61.0 \mathrm{mg} / \mathrm{L}, \mathrm{P}<0.05)$ (Fig 4B). For both strains, approximately $90 \%$ of the fatty acids were secreted into the media. Specifically, the engineered strain produced $40 \mathrm{mg} / \mathrm{L}$ of free octanoic acid and $74.9 \mathrm{mg} / \mathrm{L}$ free total fatty acids, while the control strain produced 27.7 and $55.4 \mathrm{mg} / \mathrm{L}$ of free octanoic acid and free total fatty acids, respectively. Octanoic acid accounts for about $65 \%$ molar ratio of total fatty acids with the second abundant $\mathrm{C} 16$ of $16 \%-18 \%$ (Fig. $\mathrm{S} 1)$.

In rich media, growth and glucose consumption were similar for the engineered TUFA-producing strain and the control strain (Fig 4C). The TUFA-producing strain produced a final titer of $730 \pm 40 \mathrm{mg} / \mathrm{L}$ octanoic acid and $930 \pm 30 \mathrm{mg} / \mathrm{L}$ total fatty acids, which are $29 \%$ and $16 \%$ higher than that in control strain $(620 \pm 30 \mathrm{mg} / \mathrm{L}, 800 \pm 40 \mathrm{mg} / \mathrm{L}, \mathrm{P}<0.05)$ (Fig 4D). The percent difference in the fatty acid titer between the TUFA-producing strain and control strain in rich medium is not as drastic as the difference in minimal medium. This might be due to the nutrients provided by the rich medium compensating for the damage caused by the fatty acids. Octanoic acid dominantly accounts for $76 \%-80 \%$ molar ratio of total fatty acids, with the second abundant C6 of 6\%-10\% (Fig. S1).

In conclusion, these results demonstrate the effectiveness of TUFA production in increasing octanoic acid and total fatty acids production. 

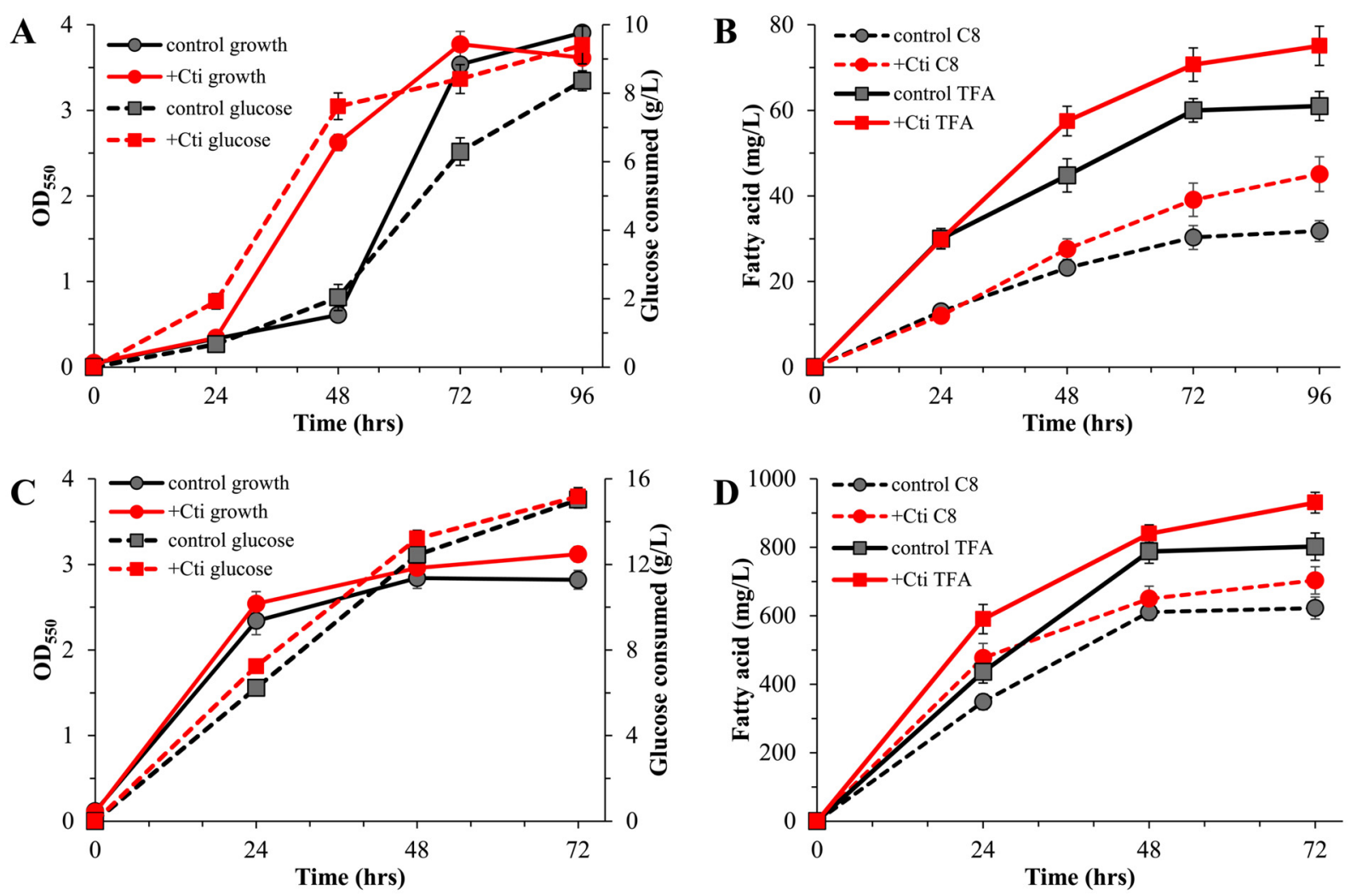

Figure 4: TUFA production enables increased carboxylic acid titers. Production of octanoic acid (C8) and total fatty acids by TUFA-producing strains in (A, B) mineral salts medium (MOPS $+2 \%(\mathrm{w} / \mathrm{v})$ dextrose) at $30{ }^{\circ} \mathrm{C}$, initial $\mathrm{pH} 7.0,120$ hours, and in $(\mathrm{C}, \mathrm{D})$ rich medium $\left(\mathrm{LB}+1.5 \%(\mathrm{w} / \mathrm{v})\right.$ dextrose) at $30{ }^{\circ} \mathrm{C}$, initial $\mathrm{pH} 7.0,72$ hours. Both the engineered and control strain were subjected to deletion of $f a d D$ to prevent fatty acid degradation and both carry the pJMYEEI82564 plasmid, which harbors thioesterase 10 (TE10). Values are the average of three biological replicates with error bars indicating one standard deviation. Control, ldhAD+TE10. Pacti, M1-12-PactiD+TE10.

\subsection{TUFA production increases tolerance to other bio-products and adverse conditions.}

Given that decreased fluidity is a general membrane characteristic change, we deemed that it should not be restricted to only improving tolerance of fatty acid. To this end, we investigated the tolerance of the M1-12-Pacti engineered strain to other inhibitory compounds and processing conditions. Four general classes of compounds were considered: other short-chain fatty acids (SCFA), alcohols, organic acids and aromatics, as well as three classes of adverse processing conditions: high temperature, low pH and high osmotic pressure (Fig 5). Each of the investigated compounds is an attractive biorenewable chemical and each of the processing conditions can impact the process cost. Concentrations for these inhibitors were selected based on previous 
reports, so that growth was at least moderately, but not completely, inhibited. For each of the compounds tested here, the specific growth rate of the control strain was decreased at least $13 \%$ but not more than $57 \%$ relative to the no-inhibitor control condition. Values for the specific growth rates are given in Table $\mathrm{S} 1$.

We found that besides octanoic acid (C8), the engineered TUFA-producing strain also showed increased tolerance to even shorter chain fatty acids. Specifically, the specific growth rate was increased by $15.0 \%$ during challenge with hexanoic acid (C6). However, TUFA production had a limited impact on tolerance to decanoic acid $(\mathrm{C} 10)$.

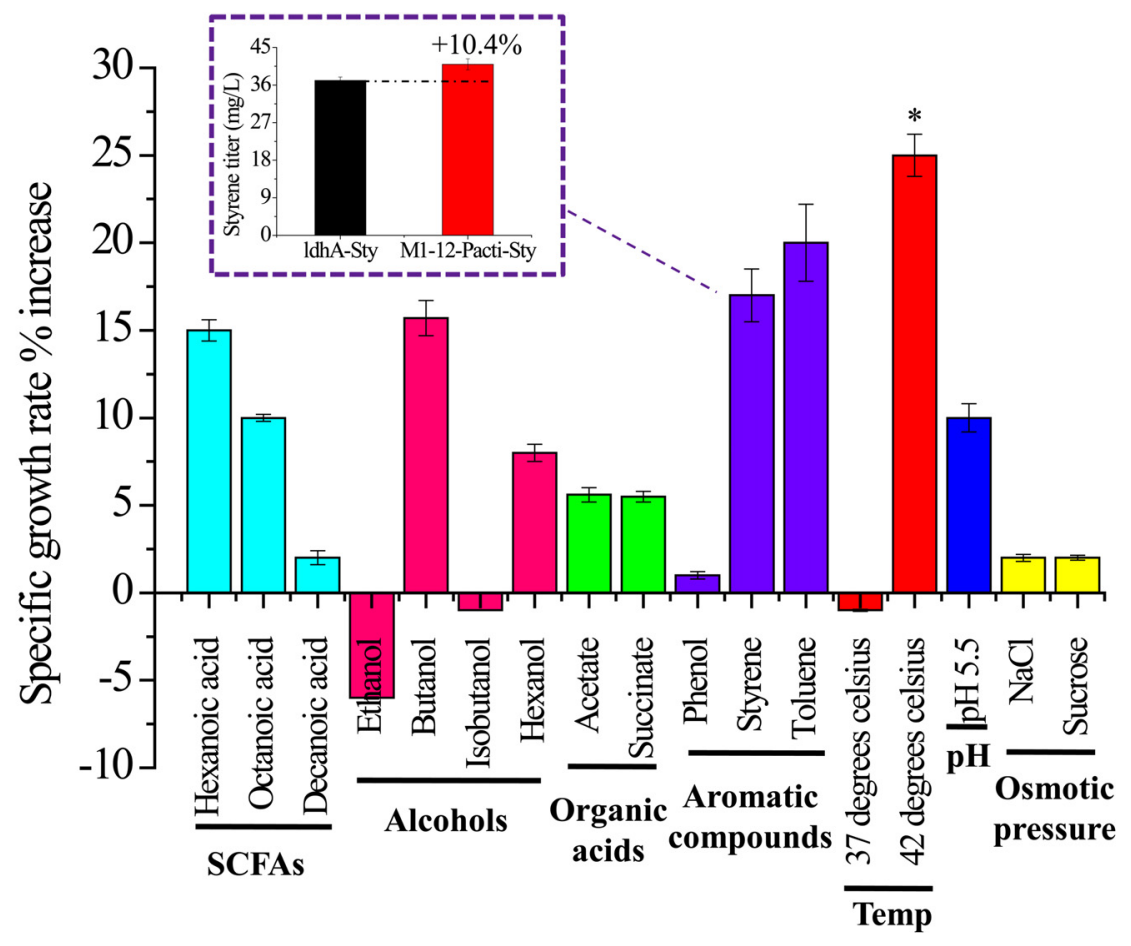

Figure 5: TUFA production enables increased tolerance to a variety of inhibitory compounds and environmental stressors.

Specific growth rate changes in the engineered TUFA-producing M1-12-Pacti strain relative to the control strain under a variety of chemical and environmental challenges. The final concentrations of each chemical are as follows. SCFA, hexanoic acid, $10 \mathrm{mM}$; octanoic acid, 10 $\mathrm{mM}$; decanoic acid, $10 \mathrm{mM}$. Alcohols: ethanol, 2\% (v/v), $343 \mathrm{mM}$; butanol, 0.6\% (v/v), $66 \mathrm{mM}$; isobutanol, $0.6 \%(\mathrm{v} / \mathrm{v}), 66 \mathrm{mM}$; hexanol, $0.1 \%(\mathrm{v} / \mathrm{v}), 8 \mathrm{mM}$. Organic acids: acetate, $30 \mathrm{mM}$; succinate, $200 \mathrm{mM}$. Aromatic compounds: phenol, $11 \mathrm{mM}$; styrene, $2 \mathrm{mM}$; toluene, $10 \mathrm{mM}$. Acid condition: initial pH 5.5. Osmotic pressure: sodium chloride, $600 \mathrm{mM}$; sucrose, $480 \mathrm{mM}$. Values are the average of at least three biological replicates with error bars indicating one standard deviation. The inset shows the styrene titer of cti engineered strain and control strain. For styrene production, M1-12-Pacti and ldhA control strains were transformed with pTpal-fdc plasmid, and styrene titer data has a P-value $<0.05$ using a two-tailed t-test. All tolerance data except under decanoic acid, isobutanol, phenol, $37^{\circ} \mathrm{C}$ conditions have a $\mathrm{P}$ value $<0.05$. Star $(*)$, cell mass $(\mathrm{g} / \mathrm{L})$ increase. SCFA, short chain fatty acids. Temp, Temperature. 
For alcohols, although the TUFA-producing strain exhibited decreased specific growth rate during challenge with either ethanol or isobutanol, it did show $16 \%$ and $8.0 \%$ increased specific growth rate during challenge with n-butanol and hexanol relative to the control, respectively. Each of these four alcohols has previously been shown to inhibit the growth of E. coli, but with distinct modes of toxicity (53-55). In general, it has been reported that the degree of membrane damage will vary due to the structure of the focal alcohol, such as the chain length and whether the alcohol is straight-chained or branched $(12,53)$.

For organic acids, there was a $5.6 \%$ increase of specific growth rate in the presence of acetate and a $5.5 \%$ increase when grown with succinate. Some hydrophobic aromatics have previously been shown to disrupt the cell membrane and are toxic to microbes (56-58). Herein, we found that although there was a limited increase in tolerance of phenol, the engineered strain had significantly enhanced tolerance to styrene $(17 \%)$ and toluene $(20 \%)(\mathrm{P}<0.05)$.

In addition to these inhibitory products, the TUFA-producing strain also had increased tolerance to harsh growth conditions. For instance, the specific growth rate in media that was at an initial $\mathrm{pH}$ of 5.5 was increased by $10 \%$. This increased acid resistance could save processing costs by decreasing the base usage to maintain a near-neutral $\mathrm{pH}$, and also decrease the vulnerability to contamination. Similarly, the engineered strain's specific growth rate at $42{ }^{\circ} \mathrm{C}$ improved by $15 \%$ relative to the non-TUFA producing control and its final cell mass increased by $25 \%$. This increased thermotolerance could be useful in industrial applications by reducing cooling water usage and decreasing the probability of contamination. Note that TUFA production showed limited impact on tolerance to high osmotic pressure conditions (Fig. 5).

In conclusion, TUFA production showed diverse effectiveness in improving tolerance to a variety of inhibitory bio-products and conditions. As with the carboxylic acid tolerance, we furthermore verified that increased tolerance of styrene enabled increased styrene production. Specifically, our TUFA-producing strain was engineered to express a previously-described pathway that converts phenylalanine to styrene (40). The control strain only contained the styrene production pathway and did not produce TUFA. We observed that in minimal medium containing phenylalanine, the TUFA-producing strain produced $10.4 \%$ more styrene than the control strain $(\mathrm{P}<0.05)$ (Fig 5, inset). These results again demonstrate the effectiveness of TUFA production on increasing both tolerance and production of biorenewable chemicals. 


\section{DISCUSSION AND CONCLUSIONS}

Product toxicity is a common obstacle for attaining sufficiently high titers of biorenewable fuels and chemicals $(59,60)$. Therefore, constructing robust organisms with high tolerance to these products is indispensable for industrial applications and has attracted extensive attention in recent years $(13,24,32,34)$. The typical approach for improving tolerance is to use evolutionary methods (61), relying on Orgel's Second Rule that "evolution is cleverer than you are". Such studies have often confirmed the fact that the membrane is an effective target for increasing robustness. For example, our own evolution of an E. coli strain with improved carboxylic acid tolerance produced a strain with significantly altered membrane integrity, fluidity and composition (34). Similarly, evolutionary work in S. cerevisiae that aimed to improve ethanol production at $40^{\circ} \mathrm{C}$ also identified critical changes in membrane composition (62).

Given this importance of the cell membrane to supporting overall metabolic behavior, the membrane has become a popular engineering target for increasing tolerance to some toxic products, including carboxylic acids or fatty acids $(14,32-34)$. Among these previous reports, many projects have focused on increasing the length of fatty acids in order to construct a "thicker" membrane with the goal of improving membrane integrity or decreasing leakage and this strategy did work in some cases (32). However, except for membrane integrity, we know little about the impact of changes in other membrane characteristics on E. coli tolerance of inhibitory compounds.

Here, we rationally sought to improve the membrane robustness by enabling homeoviscous adaptation in E. coli. Specifically, we expressed the Cti enzyme from P. aeruginosa in order to convert some of the existing CUFA to TUFA. This production of TUFA is a novel behavior for this common fermentation organism. When expression of Cti was carefully tuned, increased tolerance and production of membrane-damaging compounds was observed. The TUFA appears to primarily impact membrane function via a decrease in membrane fluidity, as opposed to increased membrane integrity that has been observed for other engineering strategies $(32,52)$. This altered membrane fluidity is consistent with the structural advantages of TUFA over CUFA and may support appropriate functioning of membrane-associated proteins during fatty production. Given the presence of a $30^{\circ}$ angle at the double bond in CUFA, the straight-chain TUFA will pack more tightly than the CUFA and thus increase membrane rigidity $(45,63)$. Presumably, a similar effect could also be seen with cis and trans forms of oleic acid (C18:1), 
though this has not been explored here.

We showed that the engineered TUFA producing strain had increased tolerance of a variety of compounds, including our primary target of octanoic acid, but also hexanoic acid, butanol, hexanol, acetate, succinate, styrene, and toluene. Robustness in industrially-attractive harsh conditions, such as high temperature $\left(42{ }^{\circ} \mathrm{C}\right)$ and low $\mathrm{pH}(5.5)$ further demonstrated the effectiveness of this membrane engineering strategy. However, despite the effectiveness of TUFA production on increasing tolerance to these adverse conditions, excessive TUFA formation was found to be counter-productive. Specifically, we found that there is a positive relationship between ratio of TUFA/CUFA and tolerance to octanoic acid when the ratio is below 0.078, while excessive TUFA formation decreased tolerance. The optimum TUFA/CUFA ratio probably differs according to the chemistry of the inhibitory molecule.

The relationship between the chemistry of a molecule, its toxicity and the effectiveness of TUFA production may be related to the hydrophobicity of the molecule. For example, when considering the octanol/water partition coefficients for each of our focal compounds, an optimal hydrophobicity appears to exist, on either side of which the efficacy of the TUFA incorporation strategy gradually diminishes (Figure 6). For example, among the organic acids tested here, TUFA production was most helpful in providing protection against hexanoic and octanoic acid (Figure 5). These two acids have $\log \mathrm{K}_{\mathrm{O} / \mathrm{w}}$ values of approximately 2 and 3, respectively. TUFA production was not protective against decanoic acid, which has a $\log \mathrm{K}_{\mathrm{O} / \mathrm{W}}$ value of 4 , or acetic acid, which has a $\log \mathrm{K}_{\mathrm{O} / \mathrm{w}}$ value of -0.2. Said optima, however, appear to differ depending on the class of compounds investigated. For organic acids, this optima is around 2; alcohols, $\sim 1$; and aromatics $>2.5$. This perhaps highlights the different mechanisms of inhibition that they elicit.

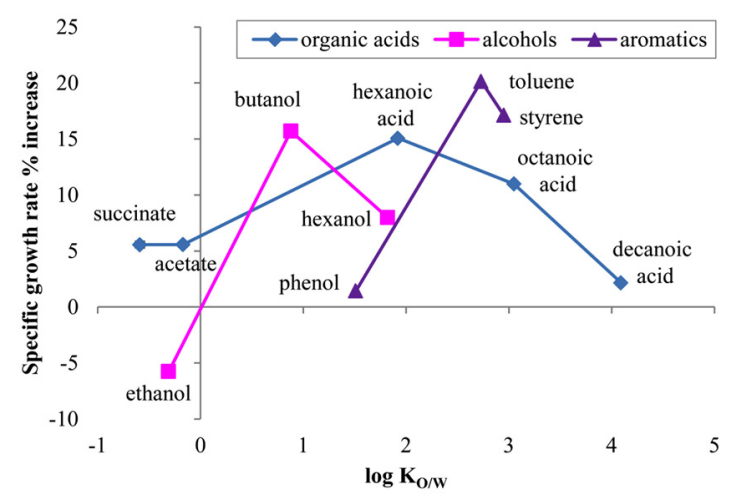

Figure 6: The protective effect of TUFA production varies according to the molecule class and octanol/water partition coefficient $\mathrm{K}_{\mathrm{O} / \mathrm{W}}$.

Although product toxicity is often identified as a primary reason for limiting further improvements in strain performance, increased tolerance does not always lead to improved 
production. In this study, our rational membrane engineering approach of enabling TUFA production not only increased tolerance to exogenously added octanoic acid but also significantly increased the octanoic acid titer $(\mathrm{P}<0.05)$. Similar results were observed in regards to styrene tolerance and styrene production. Thus, these two examples demonstrated the effectiveness of enabling TUFA production in improving biocatalyst performance.

\section{ACKNOWLEDGMENTS}

This work was supported by the NSF Engineering Research Center for Biorenewable Chemicals (CBiRC), NSF Award number EEC-0813570. David Nielsen is supported in part by NSF Award number CBET-1511637. The funders had no role in study design, data collection and analysis, decision to publish, or preparation of the manuscript. We thank Dr. Zengyi Shao for providing the Pseudomonas strain. We thank ISU Flow Cytometry Facility for help with SYTOX Green cells analysis and ISU W.M. Keck Metabolomics Research Laboratory for help with membrane polarization analysis and GC-MS analysis. 


\section{REFERENCES}

1. Gallezot P (2007) Process options for converting renewable feedstocks to bioproducts. Green Chem 9(4):295-302.

2. Larson ED (2006) A review of life-cycle analysis studies on liquid biofuel systems for the transport sector. Energy for Sustainable Development 10(2):109-126.

3. Thakker C, Martinez I, San KY, \& Bennett GN (2012) Succinate production in Escherichia coli. Biotechnology Journal 7(2):213-224.

4. Park J, et al. (2012) Synthesis of methyl ketones by metabolically engineered Escherichia coli. J Ind Microbiol Biotechnol 39(11):1703-1712.

5. McKenna R \& Nielsen DR (2011) Styrene biosynthesis from glucose by engineered E. coli. Metab Eng 13(5):544-554.

6. Zhu X, et al. (2014) Metabolic evolution of two reducing equivalent-conserving pathways for high-yield succinate production in Escherichia coli. Metab Eng 24C:87-96.

7. Atsumi S, et al. (2008) Metabolic engineering of Escherichia coli for 1-butanol production. Metab Eng 10(6):305-311.

8. Dunlop MJ (2011) Engineering microbes for tolerance to next-generation biofuels. Biotechnology for Biofuels 4.

9. Huffer S, Roche CM, Blanch HW, \& Clark DS (2012) Escherichia coli for biofuel production: bridging the gap from promise to practice. Trends in Biotechnology 30(10):538-545.

10. Yomano LP, York SW, \& Ingram LO (1998) Isolation and characterization of ethanol-tolerant mutants of Escherichia coli KO11 for fuel ethanol production. J Ind Microbiol Biot 20(2):132-138.

11. Knoshaug EP \& Zhang M (2009) Butanol Tolerance in a Selection of Microorganisms. Applied Biochemistry and Biotechnology 153(1-2):13-20.

12. Huffer S, Clark ME, Ning JC, Blanch HW, \& Clark DS (2011) Role of Alcohols in Growth, Lipid Composition, and Membrane Fluidity of Yeasts, Bacteria, and Archaea. Applied and Environmental Microbiology 77(18):6400-6408.

13. Lennen RM, et al. (2011) Membrane stresses induced by overproduction of free fatty acids in Escherichia coli. Appl Environ Microbiol 77(22):8114-8128. 
14. Liu P, et al. (2013) Membrane stress caused by octanoic acid in Saccharomyces cerevisiae. Appl Microbiol Biot 97(7):3239-3251.

15. Royce LA, Liu P, Stebbins MJ, Hanson BC, \& Jarboe LR (2013) The damaging effects of short chain fatty acids on Escherichia coli membranes. Appl Microbiol Biotechnol 97(18):8317-8327.

16. Zaldivar J \& Ingram LO (1999) Effect of organic acids on the growth and fermentation of ethanologenic Escherichia coli LY01. Biotechnol Bioeng 66(4):203-210.

17. Spector AA \& Yorek MA (1985) Membrane lipid composition and cellular function. J Lipid Res 26(9):1015-1035.

18. Trcek J, Mira NP, \& Jarboe LR (2015) Adaptation and tolerance of bacteria against acetic acid. Appl Microbiol Biotechnol 99(15):6215-6229.

19. Korstanje TJ, van der Vlugt JI, Elsevier CJ, \& de Bruin B (2015) Hydrogenation of carboxylic acids with a homogeneous cobalt catalyst. Science 350(6258):298-302.

20. Lennen RM, Braden DJ, West RM, Dumesic JA, \& Pfleger BF (2010) A Process for Microbial Hydrocarbon Synthesis: Overproduction of Fatty Acids in Escherichia coli and Catalytic Conversion to Alkanes. Biotechnology and Bioengineering 106(2):193-202.

21. Lopez-Ruiz JA \& Davis RJ (2014) Decarbonylation of heptanoic acid over carbon-supported platinum nanoparticles. Green Chem 16(2):683-694.

22. Steen EJ, et al. (2010) Microbial production of fatty-acid-derived fuels and chemicals from plant biomass. Nature 463(7280):559-U182.

23. Zhang FZ, et al. (2012) Enhancing fatty acid production by the expression of the regulatory transcription factor FadR. Metabolic Engineering 14(6):653-660.

24. Lennen RM \& Pfleger BF (2012) Engineering Escherichia coli to synthesize free fatty acids. Trends in Biotechnology 30(12):659-667.

25. Choi JW \& Da Silva NA (2014) Improving polyketide and fatty acid synthesis by engineering of the yeast acetyl-CoA carboxylase. Journal of Biotechnology 187:56-59.

26. Leber C \& Da Silva NA (2014) Engineering of Saccharomyces cerevisiae for the Synthesis of Short Chain Fatty Acids. Biotechnology and Bioengineering 111(2):347-358. 
27. Tee TW, Chowdhury A, Maranas CD, \& Shanks JV (2014) Systems Metabolic Engineering Design: Fatty Acid Production as an Emerging Case Study. Biotechnology and Bioengineering 111(5):849-857.

28. Wu H, Lee J, Karanjikar M, \& San KY (2014) Efficient free fatty acid production from woody biomass hydrolysate using metabolically engineered Escherichia coli. Bioresour Technol 169:119-125.

29. Thakker C, Martinez I, Li W, San KY, \& Bennett GN (2015) Metabolic engineering of carbon and redox flow in the production of small organic acids. J Ind Microbiol Biotechnol 42(3):403-422.

30. Lennen RM, et al. (2011) Membrane Stresses Induced by Overproduction of Free Fatty Acids in Escherichia coli. Applied and environmental microbiology 77(22):8114-8128.

31. Jarboe LR, Royce LA, \& Liu P (2013) Understanding biocatalyst inhibition by carboxylic acids. Frontiers in microbiology 4.

32. Lennen RM \& Pfleger BF (2013) Modulating Membrane Composition Alters Free Fatty Acid Tolerance in Escherichia coli. Plos One 8(1).

33. Royce LA, et al. (2014) Transcriptomic analysis of carboxylic acid challenge in Escherichia coli: beyond membrane damage. PLoS One 9(2):e89580.

34. Royce LA, et al. (2015) Evolution for exogenous octanoic acid tolerance improves carboxylic acid production and membrane integrity. Metab Eng 29:180-188.

35. Sherkhanov S, Korman TP, \& Bowie JU (2014) Improving the tolerance of Escherichia coli to medium-chain fatty acid production. Metab Eng 25:1-7.

36. Lu J, et al. (2012) Combinatorial modulation of galP and $g l k$ gene expression for improved alternative glucose utilization. Appl Microbiol Biotechnol 93(6):2455-2462.

37. Tan Z, Zhu X, Chen J, Li Q, \& Zhang X (2013) Activating phosphoenolpyruvate carboxylase and phosphoenolpyruvate carboxykinase in combination for improvement of succinate production. Appl Environ Microbiol 79(16):4838-4844.

38. Jing FY, et al. (2011) Phylogenetic and experimental characterization of an acyl-ACP thioesterase family reveals significant diversity in enzymatic specificity and activity. BMC Biochemistry 12.

39. Datsenko KA \& Wanner BL (2000) One-step inactivation of chromosomal genes in Escherichia coli K-12 using PCR products. P Natl Acad Sci USA 97(12):6640-6645. 
40. McKenna R, Pugh S, Thompson B, \& Nielsen DR (2013) Microbial production of the aromatic building-blocks (S)-styrene oxide and (R)-1,2-phenylethanediol from renewable resources. Biotechnology Journal 8(12):1465-1475.

41. Neidhard.Fc, Bloch PL, \& Smith DF (1974) Culture Medium for Enterobacteria. Journal of Bacteriology 119(3):736-747.

42. Torella JP, et al. (2013) Tailored fatty acid synthesis via dynamic control of fatty acid elongation. Proc Natl Acad Sci U S A 110(28):11290-11295.

43. Villanueva DY, Lim JB, \& Klauda JB (2013) Influence of ester-modified lipids on bilayer structure. Langmuir 29(46):14196-14203.

44. Mashaghi S, Jadidi T, Koenderink G, \& Mashaghi A (2013) Lipid nanotechnology. Int J Mol Sci $14(2): 4242-4282$.

45. Heipieper HJ, Meinhardt F, \& Segura A (2003) The cis-trans isomerase of unsaturated fatty acids in Pseudomonas and Vibrio: biochemistry, molecular biology and physiological function of a unique stress adaptive mechanism. FEMS Microbiol Lett 229(1):1-7.

46. Holtwick R, Meinhardt F, \& Keweloh H (1997) cis-trans isomerization of unsaturated fatty acids: cloning and sequencing of the cti gene from Pseudomonas putida P8. Appl Environ Microbiol 63(11):4292-4297.

47. Diefenbach R, Heipieper HJ, \& Keweloh H (1992) The Conversion of Cis into Trans Unsaturated Fatty-Acids in Pseudomonas putita P8 - Evidence for a Role in the Regulation of Membrane Fluidity. Appl Microbiol Biot 38(3):382-387.

48. Weber FJ, Ooijkaas LP, Schemen RMW, Hartmans S, \& Debont JAM (1993) Adaptation of Pseudomonas putida S12 to High-Concentrations of Styrene and Other Organic-Solvents. Applied and Environmental Microbiology 59(10):3502-3504.

49. von Wallbrunn A, Richnow HH, Neumann G, Meinhardt F, \& Heipieper HJ (2003) Mechanism of cis-trans isomerization of unsaturated fatty acids in Pseudomonas putida. J Bacteriol 185(5):1730-1733.

50. Pedrotta V \& Witholt B (1999) Isolation and characterization of the cis-trans-unsaturated fatty acid isomerase of Pseudomonas oleovorans GPo12. J Bacteriol 181(10):3256-3261.

51. Sherkhanov S, Korman TP, \& Bowie JU (2014) Improving the tolerance of Escherichia coli to medium-chain fatty acid production. Metabolic Engineering 25:1-7. 
52. Luo LH, et al. (2009) Improved ethanol tolerance in Escherichia coli by changing the cellular fatty acids composition through genetic manipulation. Biotechnology Letters 31(12):1867-1871.

53. Ingram LO \& Vreeland NS (1980) Differential effects of ethanol and hexanol on the Escherichia coli cell envelope. J Bacteriol 144(2):481-488.

54. Brynildsen MP \& Liao JC (2009) An integrated network approach identifies the isobutanol response network of Escherichia coli. Mol Syst Biol 5:277.

55. Konas RM, Daristotle JL, Harbor NB, \& Klauda JB (2015) Biophysical Changes of Lipid Membranes in the Presence of Ethanol at Varying Concentrations. The Journal of Physical Chemistry. B 119(41):13134-13141.

56. de Smet MJ, Kingma J, \& Witholt B (1978) The effect of toluene on the structure and permeability of the outer and cytoplasmic membranes of Escherichia coli. Biochim Biophys Acta 506(1):64-80.

57. Sikkema J, Debont JAM, \& Poolman B (1995) Mechanisms of Membrane Toxicity of Hydrocarbons. Microbiological Reviews 59(2):201-222.

58. Heipieper HJ, Keweloh H, \& Rehm HJ (1991) Influence of Phenols on Growth and Membrane-Permeability of Free and Immobilized Escherichia-Coli. Applied and Environmental Microbiology 57(4):1213-1217.

59. Nicolaou SA, Gaida SM, \& Papoutsakis ET (2010) A comparative view of metabolite and substrate stress and tolerance in microbial bioprocessing: From biofuels and chemicals, to biocatalysis and bioremediation. Metabolic Engineering 12(4):307-331.

60. Mukhopadhyay A (2015) Tolerance engineering in bacteria for the production of advanced biofuels and chemicals. Trends in Microbiology.

61. Jin T, Chen Y, \& Jarboe L (2016) Evolutionary Methods for Improving Production of Biorenewable Fuels and Chemicals. Biotechnologies for Biofuel Production and Optimization, (Elsevier).

62. Caspeta L, et al. (2014) Altered sterol composition renders yeast thermotolerant. Science 346(6205):75-78.

63. Zhang YM \& Rock CO (2008) Membrane lipid homeostasis in bacteria. Nature Reviews. Microbiology $6(3): 222-233$. 